\title{
DELIRIUM E TEMPO DE PERMANÊNCIA EM UNIDADE DE TERAPIA INTENSIVA
}

\author{
DELIRIUM AND LENGTH OF STAY \\ IN THE INTENSIVE CARE UNIT
}

\section{DELIRIUM Y TIEMPO DE PERMANENCIA EN LA UNIDAD DE CUIDADOS INTENSIVOS}

\author{
Ana Carolina Fáccio Azevedo ${ }^{1}$ \\ Ana Maria Silva Camargo ${ }^{2}$ \\ Elaine Machado de Oliveira ${ }^{3}$
}

\begin{abstract}
Como citar este artigo: Azevedo ACF, Camargo AMS, Oliveira EM. Delirium e tempo de permanência em Unidade de Terapia Intensiva. Rev baiana enferm. 2019;33:e-33554.

Objetivo: analisar a associação entre delirium e tempo de permanência na Unidade de Terapia Intensiva. Método: estudo de coorte prospectivo conduzido na terapia intensiva de um hospital universitário de São Paulo, Brasil, entre outubro e dezembro de 2017. Participaram todos os pacientes acima de 18 anos. As variáveis independentes foram os dados clínicos, demográficos e o tempo de permanência. A variável dependente foi o delirium, medido pelo instrumento Confusion Assessment Method (ICU). Na análise dos dados, foi realizada a estatística descritiva, correlação de Spearman, teste V Cramer e o modelo de regressão logística para o teste de associação. Resultados: o delirium foi identificado em $48,5 \%$ dos pacientes graves e correlacionado com número de doses de medicamento e tempo de permanência. A condição de saída apresentou diferença significativa entre pacientes com e sem delirium. Conclusão: o tempo de permanência aumentou em quase 10,0\% a chance de delirium na Unidade de Terapia Intensiva.
\end{abstract}

Descritores: Enfermagem. Unidades de Terapia Intensiva. Cuidados Críticos. Delirium. Tempo de Internação. Avaliação em Enfermagem.

Objective: to analyze the association between delirium and length of stay in the Intensive Care Unit. Method: prospective cohort study conducted in the intensive care unit of a university hospital of São Paulo, Brazil, between October and December 2017. The participants were all patients aged over 18 years. The independent variables were the clinical and demographic data, and the length of stay. The dependent variable was the delirium, measured by the instrument Confusion Assessment Method (ICU). Data analysis was performed with descriptive statistics, Spearman's correlation, $V$ Cramer test, and the logistic regression model to test the association. Results: delirium was identified in $48.5 \%$ of severe patients and correlated with the number of doses of medicine and length of stay. The condition of liberation showed a significant difference between patients with and without delirium. Conclusion: the length of stay increased in almost $10.0 \%$ the chance of delirium in the Intensive Care Unit.

Descriptors: Nursing. Intensive Care Units. Critical Care. Delirium. Length of Stay. Nursing Assessment.

\footnotetext{
Enfermeira. Especialista em Residência Multiprofissional em Terapia Intensiva. Pesquisadora Independente. Presidente Prudente, São Paulo, Brasil. http://orcid. org/0000-000 I-6060-2765.

2 Enfermeira. Mestre em Ciência Animal. Professora da Universidade do Oeste Paulista. Presidente Prudente, São Paulo, Brasil. amscamargo@hotmail.com. http:// orcid.org/0000-0003-4333-2542.

3 Enfermeira. Doutora em Enfermagem na Saúde do Adulto. Professora da Universidade do Oeste Paulista. Presidente Prudente, São Paulo, Brasil. elainemachado@usp.br. http://orcid.org/0000-0002-1975-297X.
} 
Objetivo: analizar la asociación entre el delirium y el tiempo de permanencia en la Unidad de Cuidados Intensivos. Método: estudio de cohorte prospectivo realizado en la unidad de cuidados intensivos de un hospital universitario de São Paulo, Brasil, entre octubre y diciembre de 2017. Participaron todos los pacientes mayores de 18 años. Las variables independientes fueron los datos clínicos, demográficos y el tiempo de permanencia. La variable dependiente fue el delirium, medido por el instrumento Confusion Assessment Method (ICU). En el análisis de datos, se utilizaron estadistica descriptiva, correlación de Spearman, prueba V de Cramer, y el modelo de regresión logistica para probar la asociación. Resultados: el delirium fue identificado en 48,5\% de pacientes graves y se correlacionó con el número de dosis de medicamento y el tiempo de permanencia. La condición de salida mostró una diferencia significativa entre los pacientes con y sin delirium. Conclusión: el tiempo de permanencia incrementó en casi 10,0\% la probabilidad de delirium en la Unidad de Cuidados Intensivos.

Descriptores: Enfermería. Unidades de Cuidados Intensivos. Cuidados Críticos. El Delirio. El Tiempo de Internación. Evaluación en Enfermería.

\section{Introdução}

O delirium é uma disfunção neurocomportamental caracterizada pelo quadro de agitação, definido como um estado confusional agudo e flutuante da consciência, da cognição e do pensamento, que pode cursar com ideias delirantes e alucinações. $\mathrm{Na}$ Unidade de Terapia Intensiva (UTI), o delirium é uma disfunção frequentemente desencadeada como consequência de fatores que podem levar a quadros de confusão ou agitação psicomotora, tais como: tratamento, gravidade clínica dos pacientes, complexidade do ambiente, medicamentos e intervenções especializadas $^{(1)}$. Estes aspectos tornam fundamental investigar sua incidência.

Em função da multiplicidade de manifestações clínicas, o delirium é classificado, de acordo com o grau de atividade psicomotora, em hipoativo, hiperativo ou misto ${ }^{(2-3)}$. As manifestações clínicas do delirium apresentam diferentes intensidades, que variam de acordo com o paciente e com os estímulos que ele recebe. O delirium hipoativo é caracterizado pelo quadro de sonolência; o delirium hiperativo pelas alterações emocionais, interpretações errôneas e desconexas com desorientação, perda da memória recente, distúrbios do ciclo sono-vigília e incapacidade de cooperar; o delirium misto apresenta variação do quadro clínico com características hipoativas e sonolência até agitação psicomotora extrema com alucinações na forma hiperativa ${ }^{(4)}$.

A hospitalização, sobretudo na UTI, caracterizada por seu ambiente complexo, fechado, tratamento com fármacos analgésicos e sedativos, comorbidades, interrupção do ciclo de sono-vigília, infecções, uso de ventilação mecânica, distúrbios metabólicos e diversas intervenções invasivas que interferem nos sentidos, compromete a percepção do ambiente, favorecendo a ocorrência do delirium ${ }^{(5)}$.

Estudos que investigaram a incidência do delirium encontraram evidência de $47 \%$ a $80 \%$ da disfunção entre pacientes graves ${ }^{(6-8)}$, que pode estar associada ao aumento do uso de sedativos e contenções mecânicas ${ }^{(9)}$. Apesar do elevado índice, o diagnóstico, com frequência, não é realizado de forma correta e compromete o tratamento e a evolução clínica dos pacientes ${ }^{(8)}$, além da dificuldade de reabilitação do déficit funcional e cognitivo, que pode comprometer a segurança ${ }^{(9)}$.

Estudos que investigaram a relação do delirium com a segurança do paciente encontraram que a falta de diagnóstico e tratamento pode levar à remoção acidental de dispositivos invasivos, como cateteres e tubos, devido à agitação psicomotora, ao maior tempo de uso de ventilação mecânica invasiva, morbidade e mortalidade do paciente ${ }^{(9-10)}$.

Outro estudo identificou ocorrência de associação entre delirium na UTI e idade em 35,4\% ${ }^{(2)}$ dos pacientes, severidade das patologias agudas, diminuição da funcionalidade e aumento do comprometimento cognitivo e da mortalidade intra-hospitalar em até quatro vezes ${ }^{(2)}$, além do 
uso de medicamentos sedativos e analgésicos. Essas circunstâncias levaram à indicação de medidas não farmacológicas como alternativa preventiva do delirium, e ao uso de escalas para a detecção precoce dessa disfunção ${ }^{(5-6,11)}$.

Outra complicação identificada em associação à ocorrência do delirium é o tempo de permanência na UTI. Quando prolongado, expõe o paciente ao maior número de procedimentos invasivos, necessários ao tratamento do paciente grave, uso de sedativos e complicações decorrentes da própria condição clínica, que impactam na percepção sensorial e cognitiva do paciente e, consequentemente, na ocorrência do delirium. Além disso, aumenta o custo do cuidado em até 39\% em relação aos pacientes que não apresentam o quadro ${ }^{(11)}$.

Estudos que investigaram a incidência do delirium e o tempo de permanência na UTI reforçam que o aumento desse tempo está associado à ocorrência do delirium ${ }^{(12-13)}$.

A identificação e o diagnóstico dessa disfunção promovem o planejamento do cuidado e de aspectos fundamentais para a evolução clínica do paciente na UTI. O tratamento precoce das manifestações clínicas impacta nos resultados dos pacientes, diminui o tempo de permanência e as complicações que podem ser decorrentes da exposição ao ambiente da UTI.

Este estudo tem como objetivo analisar a associação entre delirium e tempo de permanência na Unidade de Terapia Intensiva (UTI).

\section{Método}

Estudo do tipo coorte prospectivo conduzido na UTI geral de um hospital universitário do Oeste Paulista, São Paulo, Brasil. A unidade conta com 20 leitos para o tratamento de pacientes graves procedentes do pronto-socorro, centro cirúrgico e unidades de internação. O estudo foi desenvolvido entre os meses de setembro e novembro de 2017.

A amostra não probabilística de conveniência incluiu pacientes com 18 anos ou mais, clínicos e cirúrgicos, independente do tempo de internação, e excluiu os pacientes que apresentaram diagnóstico de demência senil e Mal de Alzheimer.

As variáveis independentes foram: sexo, faixa etária, idade, tipo de tratamento (clínico ou cirúrgico), tempo de internação na UTI, comorbidades, número de comorbidades, SAPS III ${ }^{(14)}$, uso de ventilação mecânica, uso de sedação, uso de despertar diário, diagnóstico de infecção, uso de terapia medicamentosa para o delirium, número de doses de medicamento para delirium e condição de saída da UTI (alta ou óbito). A variável dependente foi representada pelo instrumento Confusion Assessment Method-ICU (CAM-ICU), que mede a presença ou ausência do delirium.

O CAM-ICU, validado para a cultura brasileira ${ }^{(15)}$, avalia o delirium por meio de quatro dimensões: flutuação do estado mental basal, inatenção, nível de consciência alterado e pensamento desorganizado. A presença de delirium é definida quando as duas primeiras características são positivas e as duas últimas estão presentes $^{(16-17)}$.

Os dados clínicos e demográficos foram coletados nos prontuários pela enfermeira residente da Residência Multiprofissional em Terapia Intensiva. O CAM-ICU também foi rigorosamente aplicado pela enfermeira residente a cada 12 horas nos primeiros cinco dias de internação a todos os pacientes. Em caso da ausência do distúrbio, a avaliação continuou em dias alternados até a saída do paciente da UTI. Em caso de presença do delirium, a aplicação do instrumento continuou diariamente por cinco dias consecutivos ou até a sua remissão.

A análise dos dados foi realizada por meio de estatística descritiva com frequências absoluta e relativa, medidas de tendência central (médias, desvios padrão), máximo e mínimo. Para a comparação entre os grupos com e sem delirium, utilizou-se o teste V de Cramer. O teste de correlação de Spearman avaliou a correlação ente as variáveis quantitativas e presença de delirium. A força de correlação fraca mediu $0.20-0.29$, média $0.30-0.49$ e forte $\geq 0.50^{(18)}$. Para identificar a associação entre tempo de internação e presença ou ausência de delirium, utilizou-se a análise de regressão logística, com 
ajuste por meio do teste de Hosmer-Lemeshow. As análises estatísticas foram realizadas no programa Statistical Package for the Social Sciences (SPSS) 23.0, considerando a significância estatística p-valor $<0,05$.

Este estudo foi aprovado pelo Comitê de Ética em Pesquisa da instituição com Parecer n. 1355 e protocolo CAAE n. 72192717.0.0000.5515, seguindo os preceitos éticos exigidos na Resolução 466/12 do Conselho Nacional de Saúde. Quando o paciente não apresentava condições clínicas para autorizar sua participação no estudo, o contato foi realizado com o familiar ou responsável.

\section{Resultados}

A amostra foi composta por 101 pacientes, sendo que, dentre esses, 49 (48,5\%) apresentaram delirium em algum momento durante a internação na UTI.

Os pacientes com delirium apresentaram como principais condições as de origem cardiorrespiratórias, neurológicas e hematológicas.
Dentre os cirúrgicos (21,8\%), os principais motivos foram as cirurgias ortopédicas, neurológicas, gastrointestinais. Os pacientes com comorbidades apresentaram maior proporção de presença do delirium (37,6\%). Como apenas 9,9\% dos pacientes fizeram uso de medicamentos sedativos em algum momento de sua internação na UTI, a proporção do despertar diário foi baixa, tanto em pacientes que apresentaram diagnóstico positivo para delirium (8,9\%) como nos pacientes que não apresentaram delirium na UTI (5,9\%).

As principais infecções foram sepse e choque séptico (com os principais focos dos tipos pulmonar, urinário e cutâneo) em maior proporção entre os pacientes que não apresentaram delirium (16,8\%).

Em relação à conduta para o tratamento do delirium neste estudo, 36,7\% dos pacientes apresentou uso de contenção física e 11,9\%, além da contenção física, fizeram uso de medida farmacológica, sendo o haloperidol a droga de escolha utilizada em todos os casos.

Tabela 1 - Comparação de variáveis clínicas e demográficas dos pacientes, segundo presença e ausência de delirium. Presidente Prudente, São Paulo, Brasil - 2017 (N=101)

\begin{tabular}{|c|c|c|c|c|}
\hline \multirow{2}{*}{ Variáveis } & \multicolumn{2}{|c|}{ Delirium } & \multirow{2}{*}{ Total (\%) } & \multirow{2}{*}{$\mathbf{p}^{*}$} \\
\hline & $\operatorname{Sim}(\%)$ & Não (\%) & & \\
\hline Sexo & & & & 0,824 \\
\hline Feminino & 17,8 & 17,8 & 35,6 & \\
\hline Masculino & 30,7 & 33,7 & 64,4 & \\
\hline Faixa etária & & & & 0,192 \\
\hline Até 30 anos & 4,0 & 2,0 & 5,9 & \\
\hline 31 a 60 anos & 14,9 & 25,7 & 40,6 & \\
\hline 61 a 80 anos & 23,8 & 20,8 & 44,6 & \\
\hline Acima de 81 anos & 5,9 & 3,0 & 8,9 & \\
\hline Tipo de tratamento & & & & 0,369 \\
\hline Cirúrgico & 21,8 & 27,7 & 49,5 & \\
\hline Clínico & 26,7 & 23,8 & 50,5 & \\
\hline Comorbidades & & & & 0,603 \\
\hline Sim & 37,6 & 37,6 & 75,2 & \\
\hline Não & 10,9 & 13,9 & 24,8 & \\
\hline Ventilação mecânica & & & & 0,653 \\
\hline Sim & 20,8 & 19,8 & 40,6 & \\
\hline Não & 27,7 & 31,7 & 59,4 & \\
\hline Sedação & & & & 0,582 \\
\hline Sim & 9,9 & 12,9 & 22,8 & \\
\hline Não & 38,6 & 38,6 & 77,2 & \\
\hline
\end{tabular}


Tabela 1 - Comparação de variáveis clínicas e demográficas dos pacientes, segundo presença e ausência de delirium. Presidente Prudente, São Paulo, Brasil - 2017 (N=101)

\begin{tabular}{|c|c|c|c|c|}
\hline \multirow{2}{*}{ Variáveis } & \multicolumn{2}{|c|}{ Delirium } & \multirow{2}{*}{ Total (\%) } & \multirow{2}{*}{$\mathbf{p}^{*}$} \\
\hline & $\operatorname{Sim}(\%)$ & Não (\%) & & \\
\hline Despertar diário & & & & 0,335 \\
\hline Sim & 8,9 & 5,9 & 14,9 & \\
\hline Não & 39,6 & 45,5 & 85,1 & \\
\hline Infecção & & & & 0,822 \\
\hline $\operatorname{Sim}$ & 14,9 & 16,8 & 31,7 & \\
\hline Não & 33,7 & 34,7 & 68,3 & \\
\hline Condição de saída & & & & 0,004 \\
\hline Alta & 44,6 & 35,6 & 80,2 & \\
\hline Óbito & 4,0 & 15,8 & 19,8 & \\
\hline
\end{tabular}

Fonte: Elaboração própria.

* Teste V Cramer.

A Tabela 2 mostra que as comorbidades, pacientes com diagnóstico para delirium, não apesar de se apresentarem frequentes nos apresentaram significância estatística.

Tabela 2 - Comparação das comorbidades dos pacientes segundo presença e ausência de delirium. Presidente Prudente, São Paulo, Brasil - 2017. (N=101)

\begin{tabular}{|c|c|c|c|c|}
\hline \multirow[b]{2}{*}{ Comorbidades } & \multicolumn{2}{|c|}{ Delirium } & \multirow[b]{2}{*}{ Total (\%) } & \multirow[b]{2}{*}{$\mathbf{p}^{*}$} \\
\hline & $\operatorname{Sim}(\%)$ & Não (\%) & & \\
\hline Alcoolismo & & & & 0,443 \\
\hline Sim & 2,0 & 4,0 & 6,0 & \\
\hline Não & 46,5 & 47,5 & 94,0 & \\
\hline Acidente vascular cerebral & & & & 0,359 \\
\hline Sim & 4,0 & 2,0 & 5,9 & \\
\hline Não & 44,6 & 49,5 & 94,1 & \\
\hline Anemia falciforme & & & & 0,329 \\
\hline $\operatorname{Sim}$ & - & 1,0 & 1,0 & \\
\hline Não & 48,5 & 50,5 & 99,0 & \\
\hline Hepatite C & & & & 0,329 \\
\hline Sim & - & 1,0 & 1,0 & \\
\hline Não & 48,5 & 50,5 & 99,0 & \\
\hline Diabetes mellitus & & & & 0,526 \\
\hline Sim & 12,9 & 10,9 & 23,8 & \\
\hline Não & 35,6 & 40,6 & 76,2 & \\
\hline Hipertensão arterial sistêmica & & & & 0,640 \\
\hline Sim & 22,8 & 21,8 & 44,6 & \\
\hline Não & 25,7 & 29,7 & 55,4 & \\
\hline Cardiopatia & & & & 0,570 \\
\hline Sim & 4,0 & 5,9 & 9,9 & \\
\hline Não & 44,6 & 45,5 & 90,1 & \\
\hline Parkinson & & & & 0,329 \\
\hline Sim & - & 1,0 & 1,0 & \\
\hline Não & 48,5 & 50,5 & 99,0 & \\
\hline Bronquite & & & & 0,966 \\
\hline Sim & 1,0 & 1,0 & 2,0 & \\
\hline Não & 47,5 & 50,05 & 98,0 & \\
\hline
\end{tabular}


Tabela 2 - Comparação das comorbidades dos pacientes segundo presença e ausência de delirium. Presidente Prudente, São Paulo, Brasil - 2017. (N=101)

\begin{tabular}{|c|c|c|c|c|}
\hline \multirow{2}{*}{ Comorbidades } & \multicolumn{2}{|c|}{ Delirium } & \multirow{2}{*}{ Total (\%) } & \multirow[b]{2}{*}{$\mathbf{p}^{*}$} \\
\hline & $\operatorname{Sim}(\%)$ & Não (\%) & & \\
\hline Câncer & & & & 0,279 \\
\hline Sim & 3,0 & 1,0 & 4,0 & \\
\hline Não & 45,5 & 50,5 & 96,0 & \\
\hline Tabagismo & & & & 0,500 \\
\hline $\operatorname{Sim}$ & 8,9 & 6,9 & 15,8 & \\
\hline Não & 44,6 & 39,6 & 84,2 & \\
\hline Depressão & & & & 0,070 \\
\hline Sim & 3,0 & 0,0 & 3,0 & \\
\hline Não & 45,5 & 51,5 & 97,0 & \\
\hline Dislipidemia & & & & 0,141 \\
\hline Sim & 2,0 & 0,0 & 2,0 & \\
\hline Não & 46,5 & 51,5 & 98,0 & \\
\hline Hipotireoidismo & & & & 0,966 \\
\hline Sim & 1,0 & 1,0 & 2,0 & \\
\hline Não & 47,5 & 50,5 & 98,0 & \\
\hline Doença pulmonar obstrutiva crônica & & & & 0,443 \\
\hline $\operatorname{Sim}$ & 2,0 & 4,0 & 6,0 & \\
\hline Não & 46,5 & 47,5 & 94,0 & \\
\hline Drogadição & & & & 0,301 \\
\hline Sim & $1,0 \%$ & 0,0 & 1,0 & \\
\hline Não & 47,5 & 51,5 & 99,0 & \\
\hline Epilepsia & & & & 0,952 \\
\hline $\operatorname{Sim}$ & 2,0 & 2,0 & 4,0 & \\
\hline Não & 46,5 & 49,5 & 96,0 & \\
\hline Nefropatia & & & & 0,966 \\
\hline Sim & 1,0 & 1,0 & 2,0 & \\
\hline Não & 47,5 & 50,5 & 98,0 & \\
\hline Insuficiência cardíaca & & & & 0,329 \\
\hline Sim & - & 1,0 & 1,0 & \\
\hline Não & 48,5 & 50,5 & 99,0 & \\
\hline Miastenia gravis & & & & 0,329 \\
\hline Sim & - & 1,0 & 1,0 & \\
\hline Não & 48,5 & 50,5 & 99,0 & \\
\hline Obesidade & & & & 0,329 \\
\hline $\operatorname{Sim}$ & - & 1,0 & 1,0 & \\
\hline Não & 48,5 & 50,5 & 99,0 & \\
\hline Pessoas vivendo com HIV/AIDS & & & & 0,598 \\
\hline $\operatorname{Sim}$ & 3,0 & 2,0 & 5,0 & \\
\hline Não & 45,5 & 49,5 & 95,0 & \\
\hline
\end{tabular}

Fonte: Elaboração própria.

Nota: Sinal convencional utilizado:

- Dado numérico igual a zero não resultante de arredondamento.

* Teste V Cramer.

A droga de escolha para o tratamento do delirium foi o haloperidol, com o máximo de 13 doses no mesmo paciente.

Em relação ao tempo de internação dos pacientes com diagnóstico positivo para delirium, o mínimo de 2 dias foi identificado em paciente de cirurgia ortopédica e máximo de 30 dias foi identificado em paciente com disfunção respiratória. 
Tabela 3 - Medida descritiva das variáveis quantitativas. Presidente Prudente, São Paulo, Brasil - 2017 $(\mathrm{N}=101)$

\begin{tabular}{l|c|c|c|c|c}
\hline Variáveis & $\mathbf{n}$ & Média & $\begin{array}{c}\text { Desvio } \\
\text { padrão }\end{array}$ & Mínimo & Máximo \\
\hline Idade (anos) & 101 & 58 & 17 & 19 & 89 \\
Número de comorbidades & 101 & 1 & 1 & - & 3 \\
SAPS III & 101 & 53 & 22 & 21 & 105 \\
Número de doses de medicamento para & 49 & 1 & 6 & 2 & 30 \\
$\begin{array}{l}\text { delirium } \\
\text { Tempo de permanência (dias) }\end{array}$ & 101 & 7 & & \\
\hline
\end{tabular}

Fonte: Elaboração própria

Nota: Sinal convencional utilizado:

- Dado numérico igual a zero não resultante de arredondamento.

As variáveis Número de doses de medicamento para delirium $(\mathrm{p}=0,000)$ e Tempo de permanência na UTI $(\mathrm{p}=0,007)$ apresentaram correlação positiva estatisticamente significativa com a presença do delirium (Tabela 4).

Tabela 4 - Correlação entre as variáveis quantitativas dos pacientes e a presença do delirium. Presidente Prudente, São Paulo, Brasil - 2017 (N=101)

\begin{tabular}{|c|c|c|}
\hline Variáveis & Rhô & $\mathbf{p}^{*}$ \\
\hline Idade (anos) & 0,169 & 0,091 \\
\hline Número de comorbidades & 0,068 & 0,500 \\
\hline Número de doses de medicamento para delirium & 0,377 & 0,000 \\
\hline Tempo de permanência na UTI (dias) & 0,266 & 0,007 \\
\hline
\end{tabular}

Fonte: Elaboração própria.

*Teste de correlação de Spearman.

A Tabela 5 mostra aumento de quase $10 \%$ de chance de delirium $(\mathrm{p}=0,011)$ para cada dia acrescido na permanência na UTI. O ajuste do modelo realizado por meio do teste de Hosmer-Lemeshow confirmou a qualidade dos resultados $(\mathrm{p}=0,541)$.

Tabela 5 - Associação entre o tempo de permanência e delirium. Presidente Prudente, São Paulo, Brasil - $2017(\mathrm{~N}=101)$

\begin{tabular}{l|c|c|c|c|c}
\hline Variável & Estimativa & $\begin{array}{c}\text { Erro } \\
\text { padrão }\end{array}$ & $\begin{array}{c}\text { Razão de } \\
\text { chances }\end{array}$ & $\begin{array}{c}\text { Intervalo de } \\
\text { confiança 95\% }\end{array}$ & $\mathbf{p}^{*}$ \\
\hline Constante & $-0,685$ & 0,309 & 0,504 & & 0,027 \\
Tempo de internação & 0,094 & 0,037 & 1,099 & $1,022-1,182$ & 0,011 \\
\hline
\end{tabular}

Fonte: Elaboração própria.

"Regressão logística.

\section{Discussão}

A importância de identificar a incidência do delirium e sua associação com o tempo de permanência na UTI permite investigar estratégias de promoção do cuidado por meio da avaliação precoce e do tratamento das manifestações clínicas do delirium e, assim, diminuir o período de internação e a consequente exposição dos pacientes a procedimentos 
invasivos e intervenções que compõem seu tratamento intensivo.

Este estudo encontrou aumento de quase 10\% nas chances de ocorrência do delirium para cada dia acrescido no tempo de permanência na UTI. Este resultado pode ser justificado pela correlação estatisticamente significativa da presença de delirium com o tempo de permanência na unidade e com o número de doses de medicamentos para o tratamento do delirium. Neste estudo, o haloperidol foi o medicamento de escolha utilizado para o tratamento do delirium, também referido na literatura como o medicamento para a profilaxia do alto risco de delirium $^{(5,19)}$. Entretanto, os resultados mostraram que o aumento da permanência na UTI e do uso de medicamentos para o tratamento do próprio delirium reforçaram o aumento da incidência dessa manifestação.

A literatura reforça a ocorrência do delirium causada pelo aumento do tempo de permanência na UTI devido ao aumento do tempo de exposição aos fatores desencadeadores do delirium, como uso de sedativos, ventilação mecânica, intervenções terapêuticas ${ }^{(11-13)}$, déficit cognitivo preexistente, uso de drogas psicoativas, presença de dor não tratada, complicações clínicas, como insuficiência cardíaca, imobilização, hipertensão, anemia, privação de sono e sepse ${ }^{(7)}$.

As demais variáveis clínicas investigadas não apresentaram associação com a ocorrência do delirium neste estudo, mas a condição de saída da UTI apresentou diferença estatisticamente significativa em relação aos pacientes com e sem delirium. A literatura reforça que a incidência do delirium pode aumentar de três ${ }^{(7)}$ a cinco $^{(20)}$ vezes as chances de óbito. Esse resultado é decorrente de aspectos que impactam no delirium e podem levar a complicações clínicas, como doenças agudas, comorbidades e uso de terapias medicamentosas, sobretudo os benzodiazepínicos $^{(7)}$.

Apesar da ausência de associação do delirium com sedativos nesta amostra, a literatura identifica que pacientes que desevolveram delirium fizeram uso de benzodiazepínicos ${ }^{(21)}$. Os alfa2-agonistas podem ser opções melhor indicadas, por terem sido associados à diminuição em 4 dias do tempo da patologia, em comparação aos benzodiazepínicos ${ }^{(6)}$, representando estratégias poupadoras de benzodiazepínicos. Assim, diminuem o risco de delirium e de outros aspectos que também impactam na ocorrência da manifestação, como a ventilação mecânica e o tempo de permanência na $\mathrm{UTI}^{(7)}$. Esses resultados reforçam a importância de monitorizar criteriosamente a indicação de sedativos na $\mathrm{UTI}^{(21)}$.

De modo a obter um nível de sedação benéfico às condições clínicas dos pacientes, as recomendações atuais sugerem um método baseado na interrupção diária da sedação, que indica a suspensão da droga diariamente e, caso seja necessário, reinicia-se com metade da dose anterior à suspensão, avaliando a necessidade do paciente. Essa medida diminui o tempo de ventilação mecânica e de internação, com consequente redução do risco de delirium e aumento da sobrevida ao fim de um ano ${ }^{(22)}$. A relação do uso de ventilação mecânica com o delirium na UTI é justificada pelo aumento do tempo de internação e uso de sedação ${ }^{(7)}$.

Apesar da ausência de associação neste estudo, a literatura apresenta resultados da incidência do delirium em maior proporção em homens, correspondendo a $78 \%$ da amostra analisada ${ }^{(23)}$. Em relação à idade, o envelhecimento da população mundial e as mudanças na prática hospitalar identificam cada vez maior número de pacientes idosos com essa condição ${ }^{(24-25)}$. Além disso, a gravidade das patologias agudizadas impacta negativamente na funcionalidade das linhas de base e do comprometimento cognitivo ${ }^{(2)}$.

Dentre os pacientes com delirium, 37,6\% apresentam algum tipo de comorbidade e os 10,9\% que não possuem nenhum tipo de patologia prévia são jovens com diagnóstico de internação na UTI causada por trauma.

Em relação à infecção, apenas 14,9\% dos pacientes deste estudo com delirium apresentaram sepse. Apesar disso, a literatura mostra risco elevado pela disfunção neuronal causada pela presença de ocitocinas pró-inflamatórias e ativação microglial que leva à perda neuronal ${ }^{(25)}$, 
indicando a importância de aplicar a CAM-ICU para a identificação da condição clínica ${ }^{(16-17)}$.

O comprometimento prévio das funções mentais, como a presença de depressão, pode atuar como fator desencadeante e aumentar o risco para a ocorrência de delirium em pacientes de UTI, pela utilização de medicamentos e ausência de prevenção ${ }^{(23)}$.

O estudo mostra que a aplicação do instrumento CAM-ICU para identificar os sinais clínicos do delirium permite implantar medidas terapêuticas precocemente, para diminuir os riscos de aumento do tempo de internação e óbito, além de promover intervenções em aspectos que podem impactar na ocorrência dessa manifestação. Dessa forma, esse instrumento representa uma medida de intervenção terapêutica eficaz para as práticas clínicas de enfermagem e segurança do paciente.

A atuação dos profissionais de enfermagem, sobretudo do enfermeiro, é fundamental para a implantação do instrumento no controle do delirium. Medidas não farmacológicas também podem ser implantadas com a finalidade de prevenir as manifestações clínicas dessa disfunção, como a redução de ruídos e a diminuição da iluminação noturna, promover a percepção do ambiente, janelas e relógios para melhorar a orientação no tempo(5) e a flexibilização do horário de visitas, que permite maior interação com familiares e a comunicação ${ }^{(6,11)}$. Outras medidas incluem a mobilização precoce, para evitar a imobilização corporal, uso de óculos e aparelhos auditivos, promoção do sono, prevenção da hipovolemia, orientação e realização de atividades terapêuticas para o estímulo das funções cognitivas $^{(11)}$. A contenção física é outra medida não farmacológica aplicada para evitar a perda não programada de sondas, tubos, cateteres venosos, mas pode precipitar novas ocorrências de delirium e causar lesões ao paciente. Por isso, deve ser indicada com critério específico, por meio de prescrição médica ${ }^{(5)}$.

Este estudo, desenvolvido em apenas uma UTI com acompanhamento de alguns meses, apesenta como limitação o tamanho de amostra reduzido. Os resultados podem promover práticas de enfermagem seguras e impactar nos melhores resultados dos pacientes da instituição, mas não representam medidas que possam ser generalizadas.

\section{Conclusão}

O tempo de internação aumentou em quase 10,0\% a chance de delirium na UTI, que pode ter sido influenciado pelo número de doses utilizadas de haloperidol, o medicamento utilizado para o tratamento da disfunção, e pelo aumento do tempo de permanência.

\section{Colaborações:}

1 - concepção, projeto, análise e interpretação dos dados: Ana Carolina Fáccio Azevedo, Ana Maria Silva Camargo e Elaine Machado de Oliveira;

2 - redação do artigo e revisão crítica relevante do conteúdo intelectual: Ana Carolina Fáccio Azevedo, Ana Maria Silva Camargo e Elaine Machado de Oliveira;

3 - aprovação final da versão a ser publicada: Ana Maria Silva Camargo e Elaine Machado de Oliveira.

\section{Referências}

1. Mori S, Takeda JRT, Carrara FSA, Cohrs CR, Zanei SSV, Whitaker IY. Incidence and factors related to delirium in an intensive care unit. Rev esc enferm USP. 2016 Ago;50(4):585-91. DOI: http:// dx.doi.org/10.1590/S0080623420160000500014

2. Ganuza ZA, Gonzalez-Torres MÁ, Gaviria M. El Delirium: Una revisión orientada a la práctica clínica. Rev asoc esp neuropsiq [Internet]. 2012 Jun [cited 2018 Feb 12];114(32):247-59. Available from: http://scielo.isciii.es/pdf/neuropsiq/v32n114/03. pdf

3. Lamond E, Murray S, Gibson CE. Delirium screening in intensive care: A life saving opportunity. Intens crit care nurs. 2018;44:105-9. DOI: https://doi. org/10.1016/j.iccn.2017.04.014

4. Pitrowsky MT, Shinotsuka CR, Soares M, Lima MASD, Salluh JIF. The importance of delirium monitoring in the intensive care unit. Rev bras ter intensiva [Internet]. 2010 Sept [cited 2018 Feb 12];22(3):274-9. 
Available from: http://www.scielo.br/pdf/rbti/ v22n3/em_10.pdf

5. Ribeiro SCL, Nascimento ERP, Lazzari DD, Jung W, Boes AA, Bertoncello KC. Knowledge of nurses about delirium in critical patients: collective subject discourse. Texto contexto enferm. 2015 Jun;24(2):513-20. DOI: http://dx.doi. org/10.1590/0104-07072015001702014

6. Faria RSB, Moreno RP. Delirium in intensive care: an under-diagnosed reality. Rev bras ter intensive. 2013;25(2):137-47. DOI: http://dx.doi. org/10.5935/0103-507X.20130025

7. Ely EW, Margolin R, Francis J, May L, Truman B, Dittus R, et al. Evaluation of delirium in critically ill patients: Validation of the Confusion Assessment Method for the intensive Care Unit (CAM-ICU). Crit care med. 2001 Jul:29(7):1370-9. DOI: 10.1097/00003246-200107000-00012

8. Pincelli EL, Waters C, Hupsel ZN. Ações de enfermagem na prevenção do delirium em pacientes na unidade de terapia intensiva. Santa Casa medical j [Internet]. 2015 [cited 2018 Feb 12];60:131-9. Available from: http:// www.fcmsantacasasp.edu.br/images/Arquivos_ medicos/60 0/60/AR33.pdf

9. Souza RCS, Bersaneti MDR, Siqueira EMP, Meira L, Brumatti DL, Prado NRO. Nurses' training in the use of a delirium screening tool. Rev Gaúcha Enferm. 2017;38(1):e-64484. DOI: http://dx.doi. org/10.1590/1983-1447.2017.01.64484

10. Patel RP, Gambrell M, Speroff T, Scott TA, Pun BT, Okahashi J, et al. Delirium and sedation in the intensive care unit: survey of behaviors and attitudes of 1384 healthcare professional. Crit Care Med. 2009 Mar;37(3):825-32. DOI: 10.1097/ CCM.0b013e31819b8608

11. Milbrandt EB, Deppen S, Harrison PL, Shintani AK, Speroff T, Stiles RA, et al. Costs associated with delirium in mechanically ventilated patients. Crit care med. 2004 Apr;32(4):955-62. DOI: 10.1097/01. ccm.0000119429.16055.92

12. Mehta S, Cook D, Devlin JWPD, Skrobik YMD, Meade MMD, Fergusson D, et al. Prevalence, Risk Factors, and Outcomes of Delirium in Mechanically Ventilated Adults. Crit Care Med. 2015 Mar;43(3):557-66. DOI: 10.1097/ CCM.0000000000000727

13. Serafim RB, Soares M, Bozza FA, Lapa e Silva JR, Dal-Pizzol F, Paulino MC, et al. Outcomes of subsyndromal delirium in ICU: a systematic review and meta-analysis. Crit Care. 2017 Jul 12;21(1):179. DOI: 10.1186/s13054-017-1765-3

14. Le Gall JR, Lemeshow S, Saulnier F. A new simplified acute physiology score (SAPS II) based on a European/North American multicenter study. JAMA. 1993 Dec 22-29;270(24):2957-63. DOI: 10.1001/jama.270.24.2957

15. Fabbri RMA, Moreira MA, Garrido R, Almeida OP. Validity and reliability of the Portuguese version of the Confusion Assessment Method (CAM) for the detection of delirium in the elderly. Arq NeuroPsiquiatr. 2001 June;59(2A):175-9. DOI: http:// dx.doi.org/10.1590/S0004-282X2001000200004

16. Tostes ICGO, Pereira SRM, Almeida LF, Santos MM. Delirium em terapia intensiva: utilização do Confusion Assessment Method for the Intensive Care Unit pelo enfermeiro. J res: fundam care. 2018 jan/mar:10(1):2-8. DOI: 10.9789/21755361.2018.v10i1.2-8

17. Ely EW. Confusion Assessment Method for the ICU (CAM-ICU) the complete training manual [Internet]. Nashville (TN): Vanderbilt University Medical Center; 2014 [cited 2018 Feb 12]. Available from: https://uploads-ssl.webflow. aec50243a0a1e5e0c/5bad3d28b04cd592318f45cc_ The-Complete-CAM-ICU-training-manual-2016-08-31_ Final.pdf

18. Cohen J. Statistical power analysis for the social sciences. $2^{\text {nd }}$ ed. Hillsdale (NJ): LEA; 1988.

19. Van Der Boogaard M, Slooter AJC, Brüggermann RJM, Schoonhover L, Beishuizen A, Vermeijden W. Effect of Haloperidol on survival among critically ill adults with a high risk of delirium: the REDUCE randomized clinical trial. JAMA. 2018 Feb;319(7):680-90. DOI: 10.1001/jama.2018.0160

20. Schubert M, Schürch R, Boettger S, Garcia Nuñez D, Schwarz U, Bettex D, et al. A hospital-wide evaluation of delirium prevalence and outcomes in acute care patients - a cohort study. BMC Health Serv Res. 2018 Jul;18(1):550. DOI: 10.1186/ s12913-018-3345-X

21. Swart LM, Van Der Zanden V, Spies PE, Rooij SE, Van Munster BC. The comparative risk of delirium with different opioids: A systematic review. Drugs aging. 2017;34;437-43. DOI: 10.1007/ s40266-017-0455-9

22. Barr J, Fraser GL, Puntillo K, Ely EW, Gélinas C, Dasta JF, et al. Clinical practice guidelines for the management of pain, agitation, and delirium in adult patients in the intensive care unit. Crit 
care med. 2013;41(1):263-306. DOI: 10.1097/ CCM.0b013e3182783b72

23. Kanova M, Sklienka P, Kula R, Burda M, Janoutova J. Incidence and risk factors for delirium development in ICU patients - a prospective observational study. Biomed Pap Med Fac Univ Palacky Olomouc Czech Repub. 2017 Jun;161(2):187-96. DOI: 10.5507/bp.2017.004

24. Wilson J, Vincent W, Alonso Y, Murphy J, Eagan J. Delirium assessment, prevention, and treatment quality improvement initiative in adult icus. Crit Care Med. 2018;46(1):588. DOI: 10.1097/01.ccm.0000529213.97375.14

25. Van Velthuijsen E, Zwakhalen S, Mulder W, Verhey F, Kempen G. Recognition and management of delirium in older hospitalized patients. Innov Aging. 2017 Jul:1(Suppl 1):971. DOI: 10.1093/ geroni/igx004.3506

Recebido: 15 de setembro de 2019

Aprovado: 16 de dezembro de 2019

Publicado: 20 de março de 2020

A Revista Baiana de Enfermagem utiliza a Licença Creative Commons - Atribuição-NãoComercial 4.0 Internacional. https://creativecommons.org/licenses/by-nc/4.0/

Este artigo é de acesso aberto distribuído sob os termos da Licença Creative Commons (CC BY-NC).

Esta licença permite que outros remixem, adaptem e criem a partir do seu trabalho para fins não comerciais. Embora os novos trabalhos tenham de lhe atribuir o devido crédito e não possam ser usados para fins comerciais, os usuários não têm de licenciar esses trabalhos derivados sob os mesmos termos. 